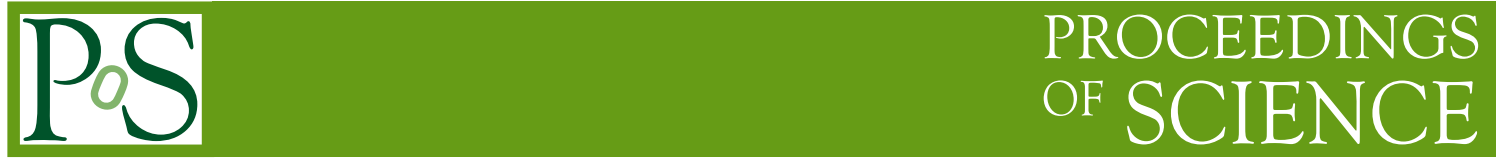

\title{
Controllability of the Gurtin-Pipkin equation
}

\author{
Luciano Pandolfi*† \\ Politecnico Di Torino \\ E-mail: luciano.pandolfi@polito.it
}

The Gurtin-Pipkin equations models the evolution of thermal phenomena when the matherial keeps memory of the past. It is well known that the Gurtin-Pipkin equation has a hyperbolic type behavior so that it is natural to expect a property of exact observability for its solutions. In this talk we consider the Gurtin-Pipkin equation with control in the Diriclet boundary condition and we prove exact controllability as a consequence of the known exact controllability of the wave equation.

Control Systems: Theory, Numerics and Applications

30 March - 1 April 2005

Rome

\footnotetext{
* Speaker.

${ }^{\dagger}$ This paper fits the programs of GNAMPA
} 


\section{Introduction}

The Gurtin-Pipkin equation, proposed in [5], is the following equation:

$$
\theta_{t}(t, x)=\int_{0}^{t} b(t-s) \Delta \theta(s, x) \mathrm{d} s+f(t, x) \quad x \in \Omega, t \geq 0 .
$$

This equation was introduced in order to model the temperature evolution in a thermal system with memory: We shall study this equation in a bounded region $\Omega$ with "regular" boundary $\Gamma$ (this assumption is discussed below). We associate an initial condition and a boundary condition of Dirichlet type to (1.1):

$$
\theta(0, x)=\theta_{0}(x) \in L^{2}(\Omega), \quad \theta(t, s)=u(s) \in L_{\mathrm{loc}}^{2}\left(0,+\infty ; L^{2}(\Gamma)\right), \quad \Gamma=\partial \Omega .
$$

Reasons for this equation: 1) According to the "usual" heat equation, a signal propagates with infinite speed. This is not physical. 2) Experience shows that at very low temperatures heat propagates in a wave-like fashion.

Both these points are addressed by Eq. (1.1) which takes into account the past history of the system. Thanks to this fact, Eq. (1.1) displays hyperbolic type properties as suggested by the special case $b \equiv 1$ and $u=0$. In this case Laplace transform gives

$$
\lambda \hat{\theta}(\lambda)-\theta_{0}=\frac{1}{\lambda} \Delta \hat{\theta}(\lambda)+\hat{f}(\lambda)
$$

which displays some "hyperbolic look". In general, it turns out that signals whose propagation is described by Eq. (1.1) have a finite speed, see [2].

Our goal is to prove that a process governed by the Gurtin-Pipkin equation (1.1) is exactly controllable in finite time, according to the following definition: there exists $T>0$ such that for every $\tilde{\theta} \in L^{2}(\Omega)$ there exists $u \in L^{2}\left(0, T ; L^{2}(\Gamma)\right)$ which drives the solution of the $G-P$ equation to hit $\tilde{\theta}$ at time $T$. In contrast with this, the heat equation is only approximately controllable.

In fact, our first goal will be to define the solutions to the G-P equation with Dirichlet boundary conditions, and to study their properties. We follow an idea of Belleni-Morante for this (see [2] and we reduce Eq. (1.1) to a Volterra equation (with bounded operators!) using the cosine operator theory.

The proofs of the results reported here can be found in [13] but the controllability proof presented here is shorter and more direct.

The bonus of this cosine operator approach is that controllability of Eq. (1.1) is a direct consequence of the (known) corresponding property of the wave equation:

$$
\begin{aligned}
& w_{t t}(t, x)=\Delta w(t, x) \quad \text { in } \Omega \\
& w(0, x)=w_{0}(x), \quad w_{t}(0, x)=w_{1}(x) \quad \text { in } \Omega \\
& w(t, s)=u(s) \text { on } \Gamma .
\end{aligned}
$$

We introduce now the assumptions used below.

Assumption 1. The kernel $b(t)$ is twice continuously differentiable and $b(0)=\mu>0$.

Controllability will be proved in section 4 under the additional assumption: 
- stronger regularity: $b \in C^{3}$;

- stronger positivity condition: $b(t)$ is integrable on $[0,+\infty)$ and

$$
\hat{b}(0)=\int_{0}^{+\infty} b(t) \mathrm{d} t>0 .
$$

The assumption that we make on the region $\Omega$ is:

Assumption 2. We assume that the region $\Omega$ is simply connected, with boundary of class $C^{2}$.

Remark 3. We observe:

- The condition $b(0)>0$ is crucial in order to have a hyperbolic type behavior. In order to simplify the notations we shall put $b(0)=1$. This amount to the choice of a suitable time scale and is not restrictive.

- The assumption on $\Omega$ is stronger then needed. For brevity we are not going to discuss this here.

It is crucial to note: under the stated assumptions on $\Omega$, the wave equation (1.3) is exactly controllable in finite time.

Finally we quote the following papers concerned with the controllability of the Gurtin-Pipkin equation: [1, 3, 10, 12]

\section{Cosine operators and the solutions of the Gurtin-Pipkin equation}

Our first step is the definition of the solutions of the Gurtin-Pipkin equation (1.1). We shall use the cosine operator theory, as presented in [4, 14] and used in [2, 7]. We shall use the same notations as in [2].

Let $A$ be the generator of an exponentially stable holomorphic semigroup on a Hilbert space $X$ (for us, the laplacian with Dirichlet homogeneous conditions on $L^{2}(\Omega)$ ). Let moreover $\mathcal{A}=$ $i(-A)^{1 / 2}$. It is known that $e^{\mathfrak{A} t}$ is a $C_{0^{-}}$group of operator on $X$. The strongly continuous operator valued function

$$
R_{+}(t)=\frac{1}{2}\left[e^{\mathscr{A} t}+e^{-\mathcal{A} t}\right] \quad t \in \mathbb{R}
$$

is called cosine operator (the cosine operator generated by $A$ ). It is convenient to introduce the operators

$$
R_{-}(t)=\frac{1}{2}\left[e^{\mathscr{A} t}-e^{-\mathcal{A} t}\right], \quad S(t)=\mathcal{A}^{-1} R_{-}(t), \quad t \in \mathbb{R} .
$$

The operator $S(t)$ is called the sine operator (generated by A.)

The following "integration by parts formulas" can be justified:

Lemma 4. Let $\xi(s) \in C^{1}(0, T ; X)$. We have

$$
\begin{aligned}
& \int_{0}^{t} R_{+}(t-s) \dot{\xi}(s) \mathrm{d} s=\xi(t)-R_{+}(t) \xi(0)+\mathcal{A} \int_{0}^{t} R_{-}(t-s) \xi(s) \mathrm{d} s \\
& \int_{0}^{t} R_{-}(t-s) \dot{\xi}(s) \mathrm{d} s=-R_{-}(t) \xi(0)+\mathcal{A} \int_{0}^{t} R_{+}(t-s) \xi(s) \mathrm{d} s
\end{aligned}
$$


Now we make some formal computations which can be justified for "very regular" data and which leads to Volterra integral equation which is then taken as the definition for the solution of the G-P equation.

We ignore for simplicity the distributed input $f(t)$.

Let $D$ be the Dirichlet map:

$$
\theta=D u \Longleftrightarrow\left\{\begin{array}{l}
\Delta \theta=0 \\
\theta_{\mid \Gamma}=u
\end{array}\right.
$$

We write Eq. (1.1) as

$$
\theta_{t}=\int_{0}^{t} b(t-s) A\{\theta(s)-D u(s)\} \mathrm{d} s .
$$

This is clearly legitimate if $\theta(t, x)$ is a classical solution of (1.1). Now, we introduce $\xi(t)=$ $\theta(t)-D u(t)$ and, if $u$ is regular and $\theta$ is a classical solution, we see that

$$
\xi_{t}=\int_{0}^{t} b(t-s) A \xi(s) \mathrm{d} s-D u^{\prime}(t)
$$

We apply the operator $R_{+}(t-s)$ to both the sides of the equality (2.3) and we integrate from 0 to $t$. After several formal manipulations (justified for "regular" data) based on the integration by parts formula we find the following integral equation for $\xi$ :

$$
\begin{aligned}
& \xi(t)=R_{+}(t) \xi(0)+\int_{0}^{t} R_{-}(t-s) \int_{0}^{s} b^{\prime}(s-r) \mathcal{A} \xi(r) \mathrm{d} r \mathrm{~d} s \\
& -D u(t)+R_{+}(t) D u(0)-\mathcal{A} \int_{0}^{t} R_{-}(t-s) D u(s) \mathrm{d} s .
\end{aligned}
$$

We re-introduce $\theta$ and we find that $\theta$ solves the following equivalent Volterra integral equations

$$
\begin{aligned}
& \theta(t)=\left\{R_{+}(t) \theta_{0}-\mathcal{A} \int_{0}^{t} R_{-}(t-s) D v(s) \mathrm{d} s\right. \\
& \left.+\int_{0}^{t} R_{+}(t-s) f(s) \mathrm{d} s\right\}+\int_{0}^{t} L(t-s) \theta(s) \mathrm{d} s, \\
& \theta(t)=\left\{R_{+}(t) \theta_{0}-\mathcal{A} \int_{0}^{t} R_{-}(t-s) D u(s) \mathrm{d} s\right. \\
& \left.-\int_{0}^{t} L(t-s) D u(s) \mathrm{d} s+\int_{0}^{t} R_{+}(t-s) f(s) \mathrm{d} s\right\} \\
& +\int_{0}^{t} L(t-s) \theta(s) \mathrm{d} s .
\end{aligned}
$$

where $u$ and $v$ are related by

$$
v(s)=u(s)+\int_{0}^{s} b^{\prime}(s-r) u(r) \mathrm{d} r .
$$

This equation admits precisely one solution $u \in L^{2}\left(0, T ; L^{2}(\Gamma)\right)$ for every $v \in L^{2}\left(0, T ; L^{2}(\Gamma)\right)$ and the transformation from $v$ to $u$ is linear, continuous and with continuous inverse, for every $T>0$. 
Remark 5. It is important now to recall that

$$
\mathcal{A} \int_{0}^{t} R_{-}(t-s) D u(s) \mathrm{d} s=A \int_{0}^{t} S(t-s) D u(s) \mathrm{d} s
$$

defines a linear and continuous transformation from $L^{2}\left(0, T ; L^{2}(\Gamma)\right)$ to $C\left(0, T ; L^{2}(\Omega)\right)$, for every $T>0$, see [8].

This important remark suggests the following definition:

Definition 6. The solutions the Gurtin-Pipkin equation (1.1) are those continuous functions $\theta(t)$ which solve the Volterra equation (2.4).

\section{Regularity properties of the solutions}

From the very definition, the solutions of Eq. (1.1) belong to $C\left([0, T], L^{2}(\Omega)\right)$. In fact, we shall need the following more precise results, which are only stated here. We recall that $\operatorname{dom} A=$ $H^{2}(\Omega) \cap H_{0}^{1}(\Omega)$ so that $\operatorname{dom} \mathcal{A}=H_{0}^{1}(\Omega)$.

Theorem 7. The transformation $\left(\theta_{0}, f, u\right) \rightarrow \theta\left(\cdot ; \theta_{0}, f, u\right)$ is linear and continuous from $L^{2}(\Omega) \times$ $L^{2}(Q) \times L^{2}(G)$ to $C\left(0, T ; L^{2}(\Omega)\right)$. If $f=0, u=0$ and $\theta_{0} \in \operatorname{dom} A^{k}$ then $\theta(t) \in C\left(0, T ; \operatorname{dom} A^{k}\right)$. Moreover,

1) If $f \in C^{1}\left(0, T ; L^{2}(\Omega)\right), u \in C^{2}\left(0, T ; L^{2}(\Gamma)\right)$ and if $\theta_{0}-D u(0) \in \operatorname{dom} \mathcal{A}$ then $\xi(t)=\theta(t)-D u(t)$ belongs to $C^{1}\left(0, T ; L^{2}(\Omega)\right) \cap C(0, T ; \operatorname{dom} \mathcal{A})$.

2) if furthermore $\theta_{0}-D u(0) \in \operatorname{dom} A, f(0)-D u^{\prime}(0)=0, f \in C^{2}\left(0, T ; L^{2}(\Omega)\right)$ and $u \in C^{3}\left(0, T ; L^{2}(\Gamma)\right)$ then: a) if $f^{\prime \prime}(t)$ and $u^{\prime \prime \prime}(t)$ are exponentially bounded, the function $\xi(t), \xi^{\prime}(t), A \xi(t)$ are continuous and exponentially bounded; b) $\theta(t)$ solves $E q$. (1.1) in the sense that $\xi(t)$ is continuously differentiable, takes values in $\operatorname{dom} A$ and for every $t \geq 0$ we have

$$
\xi_{t}=\int_{0}^{t} b(t-s) A \xi(s) \mathrm{d} s-D u^{\prime}(t)+f(t) .
$$

The results that most interest us concern normal derivatives. Let us introduce the trace operator

$$
\gamma_{1} \theta=\frac{\partial}{\partial n} \theta_{\mid \Gamma}
$$

We use the notation $b \star \theta$ to denote the convolution,

$$
(b \star \theta)(t)=\int_{0}^{t} b(t-s) \theta(s) \mathrm{d} s .
$$

Lemma 8. Let $u=0, f=0$. We have:

$$
\gamma_{1}(b \star \theta) \in H^{-1}\left(0, T ; L^{2}(\Gamma)\right)
$$

and the transformation from $\theta_{0} \in L^{2}(\Omega)$ to $\gamma_{1}(b \star \theta) \in H^{-1}\left(0, T ; L^{2}(\Gamma)\right)$ is continuous. 
The usual proofs of the controllability of wave type equations are based on two inequalities, the "direct" and the "inverse" inequality. The use of the inverse inequality for the Gurtin-Pipkin equation is bypassed here, since our controllability result is derived from the known controllability property of the wave equation. Also the direct inequality is not used. Only the weaker result in Lemma 8 is used. However, the direct inequality is an additional piece of information on the regularity of the solutions and it has its own interest. It turns out that the direct inequality holds but it needs not to be proved anew: it is a consequence of the corresponding property of the solutions of the wave equation. For completeness the proof from [13] is reported.

Let us consider the wave equation (1.3) with $u=0$ and $w_{1}=0$. It is known that

$$
w(t)=R_{+}(t) w_{0}, \quad t \in \mathbb{R}
$$

and if $w_{0} \in H_{0}^{1}(\Omega)$ then $\gamma_{1} w(t)$ exists as an element of $L^{2}\left(-T, T ; L^{2}(\Gamma)\right)$. Moreover,

$$
w_{0} \longrightarrow \gamma_{1} w(t)=\gamma_{1}\left[R_{+}(t) w_{0}\right]
$$

is continuous from $w_{0} \in H_{0}^{1}(\Omega)$ to $L^{2}\left(-T, T ; L^{2}(\Gamma)\right)$ for every $T>0$, see [9, 11, 6]. Here the full strength of the assumption made on $\Omega$ is needed.

The sense in which the trace exists is as follows: it exists for the "regular" vectors $w_{0}$. It is proved the stated continuous dependence, so that the trace operator is extended by continuity to every initial condition in $H_{0}^{1}(\Omega)$.

Theorem 9. Let $f$ and $u$ be zero. The transformation

$$
\theta_{0} \rightarrow \gamma_{1} \theta
$$

is continuous from $H_{0}^{1}(\Omega)$ to $L^{2}\left(0, T ; L^{2}(\Gamma)\right)$ for every $T>0$.

Proof. We proved (Lemma (7)) that if $\theta_{0} \in \operatorname{dom} A^{k}$ then $\theta(t) \in \operatorname{dom} A^{k}$ for every $t$ so that the trace $\gamma_{1} \theta(t)$ exists in the usual sense, provided that $k$ is large enough. We prove continuity from $H_{0}^{1}(\Omega)$ to $L^{2}(G)$ so that the transformation $\theta_{0} \rightarrow \gamma_{1} \theta(t)$ can be extended to every initial condition $\theta_{0} \in H_{0}^{1}(\Omega)$.

Let $w(t)=R_{+}(t) \theta_{0}$ be the solution of problem (1.3) (with now $w_{0}=\theta_{0}$ ). Then, $\theta(t)$ is the solution of the Volterra integral equation

$$
\begin{aligned}
& \theta(t)=w(t)+\int_{0}^{t}\left[R_{+}(t-s) b^{\prime}(0) \theta(s)+\int_{0}^{t-s} R_{+}(t-s-v) b^{\prime \prime}(v)[\theta(s)] \mathrm{d} v\right] \mathrm{d} s \\
& -\int_{0}^{t} b^{\prime}(t-s) \theta(s) \mathrm{d} s .
\end{aligned}
$$

Let $T>0$ be fixed. The properties of the wave equation recalled above show that for every fixed $s$, the function

$$
t \longrightarrow \gamma_{1}\left[R_{+}(t-s) \theta(s)\right]
$$

is square integrable, hence it is integrable. We now proceed in two steps.

Step 1) we prove that $s \rightarrow \gamma_{1}\left[R_{+}(t-s) \theta(s)\right]$ exists and belongs to $L^{2}(G)$ for a.e. $t$.

We shall prove below that the function of $s$

$$
s \rightarrow \int_{0}^{T} \gamma_{1}\left[R_{+}(t-s) \theta(s)\right] \mathrm{d} t
$$


is continuous, hence square integrable. Granted this we can integrate,

$$
\begin{aligned}
& \int_{0}^{T}\left\|\int_{0}^{T} \gamma_{1}\left[R_{+}(t-s) \theta(s)\right] \mathrm{d} t\right\|^{2} \mathrm{~d} s \\
& \leq \int_{0}^{T}\left\{T \cdot \int_{0}^{T}\left\|\gamma_{1}\left[R_{+}(t-s) \theta(s)\right]\right\|^{2} \mathrm{~d} t\right\} \mathrm{d} s<+\infty .
\end{aligned}
$$

Now, Fubini theorem shows that the function

$$
s \longrightarrow \gamma_{1}\left[R_{+}(t-s) \theta(s)\right]
$$

exists a.e. and belongs to $L^{2}(G)$. Moreover,

$$
\begin{aligned}
& \int_{0}^{T} \int_{0}^{T}\left\|\gamma_{1} R_{+}(t-s) \theta(s)\right\|^{2} \mathrm{~d} t \mathrm{~d} s=\int_{0}^{T} \int_{-s}^{T-s}\left\|\gamma_{1} R_{+}(r) \theta(s)\right\|^{2} \mathrm{~d} r \mathrm{~d} s \\
& \leq M\|\theta\|_{L^{2}\left(0, T ; H_{0}^{1}(\Omega)\right)}^{2} \leq \tilde{M}\left\|\theta_{0}\right\|_{H_{0}^{1}(\Omega)}^{2} .
\end{aligned}
$$

Inequalities (3.4) and (3.5) show that the function

$$
t \longrightarrow \int_{0}^{t} \gamma_{1}\left[R_{+}(t-s) \theta(s)\right] \mathrm{d} s
$$

as an element of $L^{2}(Q)$, depends continuously on $\theta_{0} \in H_{0}^{1}(\Omega)$.

In order to complete this argument we prove continuity of the function in (3.3). We represent

$$
\begin{aligned}
& \int_{0}^{T}\left[\gamma_{1} R_{+}(t-s) \theta(s)-\gamma_{1} R_{+}\left(t-s^{\prime}\right) \theta\left(s^{\prime}\right)\right] \mathrm{d} t \\
& =\int_{0}^{T}\left[\gamma_{1} R_{+}(t-s) \theta(s)-\gamma_{1} R_{+}(t-s) \theta\left(s^{\prime}\right)\right] \mathrm{d} t \\
& +\int_{0}^{T}\left[\gamma_{1} R_{+}(t-s) \theta\left(s^{\prime}\right)-\gamma_{1} R_{+}\left(t-s^{\prime}\right) \theta\left(s^{\prime}\right)\right] \mathrm{d} t
\end{aligned}
$$

We know from Theorem 7 point 1$)$ that if $\theta_{0} \in H_{0}^{1}(\Omega)=\operatorname{dom} \mathcal{A}$ then the solution $\theta(s)$ is continuous from $s$ to $H_{0}^{1}(\Omega)$. Hence,

$$
\lim _{s^{\prime} \rightarrow s}\left\|\theta(s)-\theta\left(s^{\prime}\right)\right\|_{H_{0}^{1}(\Omega)}=0 .
$$

This shows that the integral in (3.6) tends to zero, thanks to the regularity of the trace operator of the wave equation.

The integral in (3.7) is represented as

$$
\int_{0}^{T}\left[\gamma_{1} R_{+}\left(t-s^{\prime}+\left(s^{\prime}-s\right)\right) \theta\left(s^{\prime}\right)-\gamma_{1} R_{+}\left(t-s^{\prime}\right) \theta\left(s^{\prime}\right)\right] \mathrm{d} t .
$$

This tends to zero for $s-s^{\prime} \rightarrow 0$, thanks to the Lebesgue theorem on the continuity of the shift.

We recapitulate: we have now proved that $\gamma_{1} R_{+}(t-s) \theta(s)$ is well defined, as an element of $L^{2}$, both as a function of $t$ and as a function of $s$. In order to complete the proof:

Step 2) we prove that the trace $\gamma_{1} \theta(s)$ exists in $L^{2}(G)$ and depends continuously on $\theta_{0} \in H_{0}^{1}(\Omega)$. We go back to the equation (3.2) that we now represent as

$$
\theta(t)=F(t)+\int_{0}^{t} b^{\prime}(t-s) \theta(s) \mathrm{d} s
$$


Here

$$
F(t)=y(t)+\int_{0}^{t}\left[R_{+}(t-s) b^{\prime}(0) \theta(s)+\int_{0}^{t-s} R_{+}(t-s-v) b^{\prime \prime}(v)[\theta(s)] \mathrm{d} v\right] \mathrm{d} s
$$

and we proved that $\gamma_{1} F(t) \in L^{2}(G)$ is a continuous function of $\theta_{0} \in H_{0}^{1}(\Omega)$.

The function $b^{\prime}(t)$ is scalar, so that for $\theta_{0} \in \operatorname{dom} A^{k}, k$ large enough, we have

$$
\gamma_{1} \theta(t)=\gamma_{1} F(t)+\int_{0}^{t} k(t-s) \gamma_{1} F(s) \mathrm{d} s
$$

where $k(t)$ is the resolvent kernel of $b^{\prime}(t)$. The required continuity property of $\gamma_{1} \theta(t)$ now follows because the right hand side of this equality is a continuous function of $\gamma_{1} F(t) \in L^{2}(G)$.

\section{Controllability}

Up to now we only used $b \in C^{2}$. In fact, $b$ continuous and with two continuous derivatives defined with the exception of a finite number of points will do. For the next controllability theorem we use $b \in C^{3}$. We don't know at what extent this can be weakened.

Theorem 10. Let $b(0)>0$ and $\hat{b}(0)>0$. There exists $T>0$ and for every $\theta_{1} \in L^{2}(\Omega)$ there exists $u \in L^{2}(G)$ such that the corresponding solution $\theta(t ; u)$ of $(1.1)$ (with $f=0$ ) satisfies

$$
\theta(T ; u)=\theta_{1}
$$

In the proof we use the known results on the controllability of the wave equation and a compactness argument, and we prove first that the reachable space is closed with finite codimension for $T$ large. We then characterize the orthogonal of the reachable space and we prove that it is 0 .

Idea of the proof: let $\mathcal{L}$ be the Volterra operator in (2.4) and $\mathcal{S}$ be the i-o map of the hyperbolic system from the boundary input to the position, given by (2.6). A standard trick can be used and we can confine ourselves to the case $\|\mathcal{L}\|<q<1$ so that we have

$$
\theta=-\mathcal{S} v+\mathcal{L} \theta, \quad \theta=-[I-\mathcal{L}]^{-1} \mathcal{S} v
$$

We compute for $t=T$ and we prove that for $T$ large enough the operator $v(\cdot) \rightarrow \theta(T)$ has closed and dense image. Closure is obtained since we prove that the operator is the sum of a surjective operator (a consequence of the known controllability property of the wave equation) plus a compact operator. Thanks to this we can even say that the image has finite codimension. Density is via a direct verification, based on Lemma 8 . We present here a proof of this last fact that is easier and more direct then the proof in [13].

Let $\tilde{R}_{T}$ be the $L^{2}(\Omega)$ component of the reachable set of the wave equation:

$$
\begin{aligned}
& w_{t t}=\Delta \eta, \quad t>0, x \in \Omega \\
& w(0, x)=0, w_{t}(0, x)=0 \quad x \in \Omega, \quad w(t, x)=v(t, x) \quad \text { on } \Gamma .
\end{aligned}
$$

I.e.

$$
\tilde{R}_{T}=\left\{w(T ; v) \in L^{2}(\Omega), \quad v \in L^{2}(G)\right\}=\operatorname{Im} \mathcal{S}_{T}
$$


where

$$
\mathcal{S}_{T} v=A \int_{0}^{T} S(T-s) D v(s) \mathrm{d} s .
$$

It is known that for $T$ large we have $\tilde{\mathcal{R}}_{T}=L^{2}(\Omega)$ while we recall

$$
\theta(t ; v)=\mathcal{A} \int_{0}^{t} R_{-}(t-s) D v(s) \mathrm{d} s+\mathcal{L} \theta
$$

where

$$
\left.(\mathcal{L} \theta)(t)=\int_{0}^{t} L\right)(t-s) \theta(s) \mathrm{d} s .
$$

It is convenient to represent $\mathcal{L}=\mathcal{L}_{1}+\mathcal{L}_{2}$ where

$$
\begin{aligned}
& \left(\mathcal{L}_{1} \theta\right)(t)=\int_{0}^{t}\left[b^{\prime}(t-s) \theta(s)-\int_{0}^{t-s} R_{+}(t-s-v) b^{\prime \prime}(v)[\theta(s)] \mathrm{d} v\right] \mathrm{d} s \\
& \left(\mathcal{L}_{2} \theta\right)(t)=b^{\prime}(0) \int_{0}^{t} R_{+}(t-s) \theta(s) \mathrm{d} s .
\end{aligned}
$$

A standard trick can be used to obtain that the both the operator $\mathcal{L}_{1}, \mathcal{L}_{2}: C\left(0, T, L^{2}(\Omega)\right)$ to itself have norm less then a fixed $q<1$. We assume that this has already been

The crucial lemma that we now prove is:

Lemma 11. For every $T>0$ there exists a bounded boundedly invertible operator $J_{T}$ in $L^{2}(G)$ and a compact operator $K_{T}$ from $L^{2}(G)$ to $L^{2}(\Omega)$ such that

$$
\mathcal{R}_{T}=\operatorname{im}\left\{S_{T} J_{T}+\mathcal{K}_{T}\right\} .
$$

The condition $b \in C^{3}$ is used in order to make sense of the integration by parts needed in the proof of this lemma.

We combine this with the exact controllability of the wave equation and we get

Theorem 12. There exists $T>0$ such that the reachable set $\mathcal{R}_{T}$ is closed with finite codimension.

Moreover, due to the shift invariance of the equation, the reachable set at $T$ and we reachable set at $T+t_{0}$, obtained with inputs which are zero up to time $t_{0}$, coincide.

Now, the reachable set $\mathcal{R}(T)$ increases with time so that

$$
\mathcal{R}_{\infty}=\bigcup_{T>0} \mathcal{R}_{T}=\bigcup_{n} \mathcal{R}_{n} .
$$

We recall that for large $T$ the codimension of the reachable set is finite. This and Baire Theorem implies that the codimension of the reachable set is constant for $T$ large enough and there exists $T$ such that $\mathcal{R}_{T}=L^{2}(\Omega)$ if and only if $\mathcal{R}_{\infty}=L^{2}(\Omega)$.

Now we characterize the elements of $\left[\mathcal{R}_{\infty}\right]^{\perp}$ (a finite dimensional subspace of $L^{2}(\Omega)$ ) by using the adjoint equation

$$
\xi_{t}=-\int_{t}^{T} b(s-t) \Delta \xi(s) \mathrm{d} s, \quad \xi_{\mid \Gamma}=0, \quad \xi(T)=\xi_{0}
$$

equivalently

$$
y_{t}=\int_{0}^{t} b(t-s) \Delta y(s) \mathrm{d} s, \quad y_{\left.\right|_{\Gamma}}=0, \quad y(0)=\xi_{0}
$$


Theorem 13. The vector $\xi_{0} \in L^{2}(\Omega)$ belongs to $\left[\mathcal{R}_{T}\right]^{\perp}$ if and only if the solution $\xi(t)$ of equation (4.3) satisfies

$$
\gamma_{1} \int_{t}^{T} b(r-t) \xi(r, x) \mathrm{d} r=0 \quad 0 \leq t \leq T .
$$

equivalently if the solution $y(t)$ to Eq. (4.4) satisfies

$$
\gamma_{1} \int_{0}^{t} b(t-s) y(s, x) \mathrm{d} s=0 \quad 0 \leq t \leq T
$$

We now take a more direct route then in [13]. We want to prove that if $\xi_{0} \in R_{\infty}^{\perp}$ then $\xi=0$. We know that condition (4.6) holds for the solution of (4.4), for every $t>0$. The idea now is to take the Laplace transform of $\gamma_{1}(b \star y)$, which must be zero, and to use this to deduce that $y=0$. Unfortunately however we cannot apply an element of $H^{-1}$ to $e^{-\lambda t}$, which is not zero for $t=0$. We bypass this problem by applying $\gamma_{1}\left(b \star y\right.$ to $\left[e^{-\lambda t}-e^{-\sigma t}\right]$ where $\sigma \in \mathbb{R}$ is fixed (large enough). We first use $\xi_{n} \rightarrow \xi_{0}, \xi_{n}$ regular, so that $\gamma_{1}(b \star y)$ converges to zero in $H^{-1}$ and (the first integral denotes duality pairing and $\star$ denotes the convolution)

$$
0=\int_{0}^{+\infty}\left[e^{-\lambda t}-e^{-\sigma t}\right] \gamma_{1}\left(b(\cdot) \star y\left(\cdot ; \xi_{0}\right)\right)=\lim \int_{0}^{+\infty}\left[e^{-\lambda t}-e^{-\sigma t}\right] \gamma_{1}\left(b \star y\left(t ; \xi_{n}\right)\right) .
$$

This equality makes sense when the pairing is between $H_{0}^{1}(0, T)$ and $H^{-1}(0, T)$ but it is easy to see that the limit for $T \rightarrow+\infty$ exists provided that $\Re e \lambda$ and $\sigma \in \mathbb{R}$ are large enough.

For $\xi_{n}$ regular the second integral is easily computed

$$
\begin{aligned}
& \gamma_{1}\left\{\hat{b}(\lambda)[\lambda I-\hat{b}(\lambda) A]^{-1} \xi_{n}-\hat{b}(\sigma)[\sigma I-\hat{b}(\sigma) A]^{-1} \xi_{n}\right\} \\
& =\left[\frac{\sigma}{\hat{b}(\sigma)}-\frac{\lambda}{\hat{b}(\lambda)}\right] \gamma_{1}\left\{\left(\frac{\lambda}{\hat{b}(\lambda)} I-A\right)^{-1}\left[\frac{\sigma}{\hat{b}(\sigma)} I-A\right]^{-1} \xi_{n} .\right\}
\end{aligned}
$$

It is known that $\gamma_{1}$ when applied to elements of the domain of $A$ is given by $-D^{*} A$ so that the

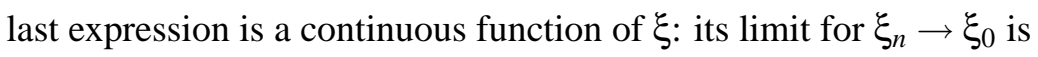

$$
\left[\frac{\sigma}{\hat{b}(\sigma)}-\frac{\lambda}{\hat{b}(\lambda)}\right] \gamma_{1}\left\{\left(\frac{\lambda}{\hat{b}(\lambda)} I-A\right)^{-1}\left[\frac{\sigma}{\hat{b}(\sigma)} I-A\right]^{-1} \xi_{0} \cdot\right\}
$$

and, as we noted, this is zero. Hence we have

$$
\gamma_{1}\left(\frac{\lambda}{\hat{b}(\lambda)} I-A\right)^{-1} \Xi=0, \quad \Xi=[\sigma I-\hat{b}(\sigma) A]^{-1} \xi_{0} \in \operatorname{dom} A .
$$

This equality holds for every $\lambda \in C$ with the exception of a discrete set, where $\lambda / \hat{b}(\lambda)$ takes values in the spectrum of $A$ and a suitable but fixed value of $\sigma$.

We recall that $\hat{b}(0)>0$ and $\hat{b}(\lambda)$ is real for $\lambda$ real. Hence, any positive $z^{2}$ (close to zero) has the form $\lambda / \hat{b}(\lambda)$ for $\lambda$ on a suitable interval of the real axis. It follows that for such values of $z$, hence for every $z$ by analytic continuation, we have

$$
\gamma_{1}\left(z^{2} I-A\right)^{-1} \Xi_{0}=0
$$

Now, $\hat{w}(\lambda)=\left(z^{2} I-A\right)^{-1} \Xi_{0}$ is the Laplace transform of the solution $w(t)$ of the wave equation (1.3) with 
- homogeneous Dirichlet conditions: $\gamma_{0} w(t)=0$ where $\gamma_{0}$ is the trace on $\Gamma$ since $w(0)=\Xi_{0} \in$ $\operatorname{dom} A, w_{t}(0)=0$.

- and, at the same time, with $\gamma_{1} w(t)=0$.

From [11, Cap. I, Cor. 5.1] we see that $w(t)=0$, hence $\Xi=0$ so that also $\xi_{0}=0$, as wanted.

\section{References}

[1] V. Barbu, M. Iannelli, Controllability of the heat equation with memory, Diff Integral Eq 200213 1393-1412.

[2] A. Belleni-Morante, An integro-differential equation arising from the theory of heat conduction in rigid materials with memory, Boll Unione Mat Ital 1978 (5) 15-B 470-482.

[3] B. Benabdallah, M.G. Naso, Null controllability of a thermoelastic plate, Abstr Appl Anal (2000) 7:585-599.

[4] H.O. Fattorini, Second order linear differential equations in Banach spaces, North-Holland, Notas de Matemàtica (99), Amsterdam, 1985.

[5] M.E. Gurtin ME, A.G. Pipkin, A general theory of heat conduction with finite wave speed. Arch. Rat. Mech. Anal. 1968 31:113-126.

[6] V. Komornik, Exact controllability and stabilization: the multiplier method, Masson, Paris, 1994.

[7] I. Lasiecka, R. Triggiani, A cosine operator approach to $L_{2}\left(0, T ; L_{2}(\Gamma)\right)$-boundary input hyperbolic equations, Appl. Mat. Optim. 19817 35-93.

[8] I. Lasiecka, R. Triggiani, ) Regularity of hyperbolic equations under $L_{2}\left(0, T ; L_{2}(\Gamma)\right)$-Dirichlet boundary terms, Appl. Mat. Optim. 198310 275-286.

[9] I. Lasiecka, R. Triggiani, Exact boundary controllability on $L_{2}(\Omega) \times H^{-1}(\Omega)$ of the wave equation with Dirichlet boundary control acting on a portion of the boundary $\partial \Omega$ and related problems. Appl. Mat. Optim. 198818 241-277.

[10] G. Leugering, Time optimal boundary controllability of a simple linear viscoelastic liquid. Math. Meth. Appl. Sci. 1987 9:413-430.

[11] J.L.Lions, Contrôlabilitè exacte, perturbations et stabilization de systémes distribuès, Vol. 1, Masson, Paris, 1988.

[12] J.E. Muñoz Rivera, M.G. Naso, (2003) Exact boundary controllability in thermoelasticity with memory. Adv. Diff. Eq. 20038 471-490.

[13] L. Pandolfi, The controllability of the Gurtin-Pipkin equation: a cosine operator approach, Applied Mathematics and Optimization to appear.

[14] M. Sova, Cosine operator functions. Rozprawy Mat 1966, 49 1-47. 